\title{
Diseño y validación de un nomograma pronóstico de supervivencia en pacientes con tumores neuroendocrinos primarios del apéndice cecal
}

\author{
Design and validation of a prognostic survival nomogram in patients \\ with primary neuroendocrine tumors of the cecal appendix
}

\author{
Andrea Carolina Quiroga-Centeno1 ${ }^{1}$ Carlos Augusto Quiroga-Centeno², \\ Juan Paulo Serrano Pastrana ${ }^{3}$, Sergio Alejandro Gómez-Ochoa ${ }^{4} \mathbb{D}$
}

1 Médico, residente de Cirugía general, Universidad Industrial de Santander, Bucaramanga, Colombia.

2 Estudiante de Medicina, Universidad Industrial de Santander, Bucaramanga, Colombia.

3 Médico, especialista en Cirugía general, jefe del Departamento de Cirugía, Universidad Industrial de Santander, Bucaramanga, Colombia.

4 Médico, MSc en Bioinformática y bioestadística. Grupo de Estudios en Salud pública y epidemiología, Fundación Cardiovascular de Colombia, Floridablanca, Colombia.

Trabajo ganador del primer puesto en el Concurso de Investigación en Cirugía "José Félix Patiño Restrepo" del Congreso Semana Quirúrgica Nacional 2020 de la Asociación Colombiana de Cirugía. Bogotá, D.C, Colombia. Noviembre de 2020.

\section{Resumen}

Introducción. Los tumores neuroendocrinos apendiculares (TNE-A) corresponden a la neoplasia apendicular más común. Aunque habitualmente tienen un comportamiento benigno, su potencial de extensión regional y metástasis, hacen necesario determinar de manera precisa el pronóstico de cada paciente. El objetivo del presente estudio fue diseñar y validar un nomograma pronóstico para predecir la supervivencia de los pacientes con TNE-A.

Métodos. Estudio de cohorte retrospectiva, de acuerdo a la información de la base de datos de vigilancia, epidemiología y desenlaces del Instituto Nacional de Cáncer de los Estados Unidos de América. Se incluyeron los pacientes con diagnóstico de TNE-A entre 1978 y 2016. El análisis de supervivencia se realizó mediante un modelo de regresión de Cox. Con estos resultados se construyeron los nomogramas para la supervivencia general y específica de cáncer a uno, dos, tres y cinco años. Los análisis fueron realizados en el software estadístico R (v. 3.5.3).

Resultados. Se incluyeron 3585 pacientes con diagnóstico de TNE-A, el 55,8 \% fueron mujeres, y la mediana de edad fue de 49 años. El subtipo histológico más frecuente fue el Tumor de Histología Mixta (THM). La edad, el subtipo histológico, el tamaño y la extensión tumoral, fueron las únicas variables asociadas independientemente con la supervivencia después del análisis multivariado. El nomograma validado presentó una capacidad de discriminación sobresaliente para predecir tanto supervivencia general 0,81 ( IC $_{95 \%}$ : 0,76-0,86), como específica a cáncer 0,88 (IC $95 \%$ : 0,83 a 0,92).

Fecha de recibido: 15/09/2020 - Fecha de aceptación: 14/12/2020 - Fecha de publicación en línea: 05/03/2021

Correspondencia: Andrea Carolina Quiroga-Centeno, Carrera 33 \# 28-126, Hospital Universitario de Santander, Bucaramanga, Colombia. Teléfono: 3005688335. Correo electrónico: caroline_aqc@hotmail.com.

Citar como: Quiroga-Centeno AC, Quiroga-Centeno CA, Serrano Pastrana JP, Gómez-Ochoa SA. Diseño y validación de un nomograma pronóstico de supervivencia en pacientes con tumores neuroendocrinos primarios del apéndice cecal. Rev Colomb Cir. 2021;36:221-36. https://doi.org/10.30944/20117582.836

Este es un artículo de acceso abierto bajo una Licencia Creative Commons - BY-NC-ND https://creativecommons.org/licenses/by-nc$\mathrm{nd} / 4.0 /$ deed.es 
Discusión. El presente estudio propone un nomograma pronóstico de supervivencia para pacientes con TNE-A, teniendo en cuenta el subtipo histológico, y alcanza una capacidad de discriminación sobresaliente para la predicción de estos desenlaces. Destacamos el peor pronóstico de los pacientes con THM, además de la supervivencia similar entre los pacientes llevados a hemicolectomía y aquellos sometidos a apendicectomía o resección del ciego, luego del análisis multivariado. Se requiere evaluar el rol de modalidades terapéuticas adyuvantes en la supervivencia de estos pacientes.

Palabras clave: nomograma; tumores neuroendocrinos; neoplasias del apéndice; histología; pronóstico; supervivencia.

\begin{abstract}
Introduction. Neuroendocrine tumors of the appendix (NET-A) correspond to the most common appendicular neoplasia. Although they usually have a benign behavior, their potential for regional extension and metastasis makes it necessary to accurately determine the prognosis of each patient. The objective of the present study was to design and validate a prognostic nomogram to predict survival of patients with NET-A.

Methods. Retrospective cohort study, based on information from the surveillance, epidemiology, and outcomes database of the National Cancer Institute of the United States of America. Patients diagnosed with NET-A between 1978 and 2016 were included. Survival analysis was performed using a Cox regression model. With these results, nomograms for general and cancer-specific survival at one, two, three and five years were constructed. The analyzes were carried out in the statistical software R (v. 3.5.3).

Results. 3585 patients with a NET-A diagnosis were included, 55.8\% were women, and the median age was 49 years. The most frequent histological subtype was the Mixed Histology Tumor (MHT). Age, histological subtype, size and tumor extension were the only variables independently associated with survival after multivariate analysis. The validated nomogram presented an outstanding discrimination capacity to predict both overall survival 0.81 ( $95 \%$ CI: 0.76-0.86) and cancer specific survival 0.88 (95\% CI: 0.83 to 0.92 ).

Discussion. The present study proposes a prognostic survival nomogram for patients with NET-A, taking into account the histological subtype, and achieves an outstanding discrimination capacity for the prediction of these outcomes. We highlight the poorer prognosis of patients with MHT, in addition to the similar survival between patients undergoing hemicolectomy and those undergoing appendectomy or resection of the cecum, after multivariate analysis. It is necessary to evaluate the role of adjuvant therapeutic modalities in the survival of these patients.
\end{abstract}

Keywords: nomogram; neuroendocrine tumors; neoplasms of the appendix; histology; prognosis; survival.

\section{Introducción}

Los tumores neuroendocrinos (TNE) son neoplasias de manifestación clínica y comportamiento variados, que se originan en las células del sistema neuroendocrino difuso ${ }^{1}$. Su lugar de presentación se asocia con tejidos derivados del intestino primitivo, y el $42 \%$ de estos tumores se localizan en el intestino medio incluyendo intestino delgado y apéndice ${ }^{2,3}$. Aunque menos frecuentes que otras neoplasias malignas, su incidencia y prevalencia han aumentado progresivamente, lo cual se atribuye a la implementación de estudios de tamización que permiten la detección de la enfermedad en estadios más tempranos ${ }^{4}$.

Los TNE son el tipo de neoplasia apendicular más común y usualmente se encuentran de forma incidental posterior a apendicectomías. La mayoría de los tumores neuroendocrinos apendiculares (TNE-A), se localizan en la punta del apéndice y son usualmente menores de $2 \mathrm{~cm}$ de diámetro ${ }^{5}$. Si 
bien, estos tumores se caracterizan habitualmente por un curso clínico indolente y un comportamiento benigno, tienen potencial de extensión regional (25-50 \%) y metástasis a distancia (10 \%), y el riesgo de metástasis ganglionares aumenta dramáticamente con tamaños tumorales mayores de $2 \mathrm{~cm}$ y con la mayor profundidad de la invasión ${ }^{2,6}$. En el apéndice se pueden encontrar además de los TNE, casi de forma exclusiva, los tumores de histología mixta (THM) que se caracterizan por contener tanto elementos neuroendocrinos como glandulares. Dentro de este subtipo histológico, el tumor carcinoide de células caliciformes (TCCC) es la neoplasia más frecuente. Los TCCC representan el 14-19\% de las neoplasias apendiculares primarias y generalmente tienen un comportamiento más agresivo que los demás TNE-A, el cual puede variar de acuerdo con la proporción del componente glandular dentro del tumor ${ }^{7}$.

Debido a la variedad en el comportamiento de los TNE-A, es necesario determinar los factores involucrados en el pronóstico de los pacientes con estas neoplasias y sus variantes, y basados en ellos, establecer el pronóstico individual de cada paciente, que permita definir las estrategias quirúrgicas y oncológicas más adecuadas para cada caso.

Un nomograma es una representación gráfica de un modelo estadístico que sirve para predecir pronóstico en pacientes con cáncer, estableciendo la probabilidad individual de un evento clínico, mediante la integración de diversas variables predictoras ${ }^{8}$. Hasta el momento, en la literatura no existen nomogramas de pronóstico para los TNE-A, por eso, nuestro objetivo fue desarrollar un nomograma clínico-patológico con el fin de predecir la supervivencia de estos pacientes a 1 , 2, 3 y 5 años.

\section{Métodos}

Este es un estudio de cohorte retrospectiva, en el cual se analiza la información disponible en la base de datos de vigilancia, epidemiología y desenlaces (Surveillance, Epidemiology, and End Results [SEER, por sus siglas en inglés]), del Instituto Nacional de Cáncer de los Estados Unidos de América.

\section{Base de datos y criterios de elegibilidad}

Para la obtención de la información del presente estudio utilizamos el software SEER*Stat versión 8.3.6.1 (https://seer.cancer.gov/seerstat/). Se filtró inicialmente por sitio primario del tumor, incluyendo únicamente aquellos que tuvieran como origen el apéndice cecal, de acuerdo con los códigos según la International Classification of Diseases (ICD): C18.1-Appendix. Posteriormente, se filtró de acuerdo con la clasificación histopatológica del tumor, incluyendo a aquellos compatibles con tumores neuroendocrinos (Código ICD-O-3: 8240/3: Carcinoid tumor, NOS, 8245/3: Adenocarcinoid tumor, 8243/3: Goblet cell carcinoid, 8244/3: Mixed adenoneuroendocrine carcinoma, 8013/3: Large cell neuroendocrine carcinoma, 8241/3: Enterochromaffin cell carcinoid, 8246/3: Neuroendocrine carcinoma, NOS, 8249/3: Atypical carcinoid tumor).

A partir de los resultados obtenidos se incluyeron solo aquellos registros con una confirmación histopatológica de la neoplasia, un seguimiento completo y la disponibilidad de la causa de muerte en el registro. Finalmente, fueron excluidos aquellos pacientes en los que el tumor neuroendocrino del apéndice cecal no representaba el tumor primario, aquellos en los que no existían datos sobre las características del tumor (tamaño, grado histológico o extensión) y aquellos que no fueron llevados a cirugía.

\section{Variables evaluadas}

Se incluyeron inicialmente variables clínicopatológicas, destacando la edad en el momento del diagnóstico, el sexo, la raza, el subtipo histológico, el grado de diferenciación celular, el tamaño y el estadio tumoral. Adicionalmente, se registró el tratamiento recibido (quimioterapia, radioterapia y manejo quirúrgico), y los datos de supervivencia (supervivencia en meses y desenlace vital).

La edad al momento del diagnóstico se clasificó como menor de 53, 53-80 y 80 años o más, según los resultados obtenidos por el software X-tile (véase en la sección de análisis estadístico). La raza se clasificó como blanca, afrodescendiente y otras (india americana/nativa de Alaska, asiática/isleña del Pacífico). El 
grado histológico se clasificó teniendo en cuenta las categorías de la variable "Grado ICD-0-3", siendo divididas en dos grupos: Grados I-II y III-IV. El tamaño del tumor se clasificó en base a su eje más largo $(<1 \mathrm{~cm}, 1-2 \mathrm{~cm}, 2-5 \mathrm{~cm}, 5-10$ $\mathrm{cm}$ y $>10 \mathrm{~cm}$ ). Los subtipos histológicos fue- ron asignados de acuerdo a la clasificación de la OMS como tumores neuroendocrinos bien diferenciados (TNEBD), carcinomas neuroendocrinos pobremente diferenciados (CNEPD) y tumores de histología mixta (THM) como se puede observar en la Tabla 1.

Tabla 1. Características demográficas y clínicas de los pacientes con tumores neuroendocrinos del apéndice cecal de acuerdo con el grupo de estudio.

\begin{tabular}{|c|c|c|c|c|}
\hline & $\begin{array}{l}\text { Grupo de validación } \\
\qquad(n=1408)\end{array}$ & $\begin{array}{l}\text { Grupo de entrenamiento } \\
\qquad(n=2177)\end{array}$ & $\begin{array}{c}\text { Total } \\
(n=3585)\end{array}$ & Valor de $p$ \\
\hline Edad (Años) & & & & 0,471 \\
\hline Media (SD) & $46,6(17,7)$ & $47,0(17,5)$ & $46,8(17,6)$ & \\
\hline Mediana (Q1, Q3) & $48(33,60)$ & $49(33,60)$ & $49(33,60)$ & \\
\hline Mínima - Máxima & $8,0-94,0$ & $4,0-92,0$ & $4,0-94,0$ & \\
\hline Sin dato & 0 & 0 & 0 & \\
\hline Edad (Categorías) & & & & 0,845 \\
\hline 0 - 52 años & $867(61,6 \%)$ & $1329(61,0 \%)$ & $2196(61,3 \%)$ & \\
\hline 53 - 80 años & $511(36,3 \%)$ & $806(37,0 \%)$ & $1317(36,7 \%)$ & \\
\hline 80 - 120 años & $30(2,1 \%)$ & $42(1,9 \%)$ & $72(2,0 \%)$ & \\
\hline Sin dato & 0 & 0 & 0 & \\
\hline Sexo & & & & 0,305 \\
\hline Mujer & $771(54,8 \%)$ & $1230(56,5 \%)$ & $2001(55,8 \%)$ & \\
\hline Hombre & $637(45,2 \%)$ & $947(43,5 \%)$ & $1584(44,2 \%)$ & \\
\hline Sin dato & 0 & 0 & 0 & \\
\hline Raza & & & & 0,751 \\
\hline Afrodescendiente & $107(7,7 \%)$ & $181(8,4 \%)$ & $288(8,1 \%)$ & \\
\hline Blanco & $1225(88,2 \%)$ & $1882(87,5 \%)$ & $3107(87,8 \%)$ & \\
\hline Otro & $57(4,1 \%)$ & $88(4,1 \%)$ & $145(4,1 \%)$ & \\
\hline Sin dato & 19 & 26 & 45 & \\
\hline Estado marital & & & & 0,606 \\
\hline Casado & $724(54,4 \%)$ & $1096(53,5 \%)$ & $1820(53,8 \%)$ & \\
\hline Soltero & $607(45,6 \%)$ & $953(46,5 \%)$ & $1560(46,2 \%)$ & \\
\hline Sin dato & 77 & 128 & 205 & \\
\hline Grado histológico & & & & 0,298 \\
\hline Grado I-II & $698(81,5 \%)$ & $1022(79,7 \%)$ & $1720(80,4 \%)$ & \\
\hline Grado III-IV & $158(18,5 \%)$ & $260(20,3 \%)$ & $418(19,6 \%)$ & \\
\hline Sin dato & 552 & 895 & 1447 & \\
\hline Subtipo histológico & & & & 0,102 \\
\hline TNEBD & $533(43,8 \%)$ & $789(41,1 \%)$ & $1322(42,1 \%)$ & \\
\hline THM & $617(50,7 \%)$ & 1017 (52,9 \%) & $1634(52,1 \%)$ & \\
\hline CNEPD & $68(5,6 \%)$ & $115(6,0 \%)$ & $183(5,8 \%)$ & \\
\hline Sin dato & 17 & 23 & 40 & \\
\hline Tamaño tumoral & & & & 0,275 \\
\hline
\end{tabular}




\begin{tabular}{|c|c|c|c|c|}
\hline & $\begin{array}{l}\text { Grupo de validación } \\
(n=1408)\end{array}$ & $\begin{array}{c}\text { Grupo de entrenamiento } \\
(n=2177)\end{array}$ & $\begin{array}{c}\text { Total } \\
(n=3585)\end{array}$ & Valor de $p$ \\
\hline$<1 \mathrm{~cm}$ & $429(33,5 \%)$ & $682(35,3 \%)$ & $1111(34,6 \%)$ & \\
\hline $1-2 \mathrm{~cm}$ & $240(18,7 \%)$ & $324(16,81 \%)$ & $564(17,6 \%)$ & \\
\hline $2-5 \mathrm{~cm}$ & $524(41,0 \%)$ & $784(40,6 \%)$ & $1308(40,8 \%)$ & \\
\hline $5-10 \mathrm{~cm}$ & $77(6,0 \%)$ & $133(6,9 \%)$ & $210(6,5 \%)$ & \\
\hline$>10 \mathrm{~cm}$ & $7(0,7 \%)$ & $4(0,2 \%)$ & $11(0,4 \%)$ & \\
\hline Sin dato & 371 & 574 & 945 & \\
\hline Extensión & & & & 0,599 \\
\hline Distante & $127(9,1 \%)$ & $178(8,3 \%)$ & $305(8,6 \%)$ & \\
\hline Regional & $359(25,8 \%)$ & $546(25,3 \%)$ & $905(25,5 \%)$ & \\
\hline Localizada & $906(65,1 \%)$ & $1431(66,4 \%)$ & $2337(65,9 \%)$ & \\
\hline Sin dato & 16 & 22 & 38 & \\
\hline Radioterapia & & & & 0,843 \\
\hline No & $1404(99,7 \%)$ & $2170(99,7 \%)$ & $3574(99,7 \%)$ & \\
\hline $\mathrm{Si}$ & $4(0,3 \%)$ & $7(0,3 \%)$ & $11(0,3 \%)$ & \\
\hline Sin dato & 0 & 0 & 0 & \\
\hline Quimioterapia & & & & 0,236 \\
\hline No & $1266(89,9 \%)$ & $1930(88,7 \%)$ & $3196(89,1 \%)$ & \\
\hline $\mathrm{Si}$ & $142(10,1 \%)$ & $247(11,3 \%)$ & $389(10,9 \%)$ & \\
\hline Sin dato & 0 & 0 & 0 & \\
\hline Cirugía & & & & 0,637 \\
\hline $\begin{array}{l}\text { Apendicectomía o } \\
\text { resección de ciego }\end{array}$ & $838(59,5 \%)$ & $1263(58,0 \%)$ & $2101(58,6 \%)$ & \\
\hline Colectomía subtotal & $523(37,1 \%)$ & $843(38,7 \%)$ & $1366(38,1 \%)$ & \\
\hline Colectomía total & $47(3,3 \%)$ & $71(3,3 \%)$ & $118(3,3 \%)$ & \\
\hline Sin dato & 0 & 0 & 0 & \\
\hline Mortalidad General & & & & 0,554 \\
\hline No & $1179(83,7$ \%) & $1839(84,5 \%)$ & $3018(84,2 \%)$ & \\
\hline $\mathrm{Si}$ & $229(16,3 \%)$ & $338(15,5 \%)$ & $567(15,8 \%)$ & \\
\hline Sin dato & 0 & 0 & 0 & \\
\hline \multicolumn{2}{|c|}{ Mortalidad Asociada a Cáncer } & & & 0.494 \\
\hline No & $1253(89,0 \%)$ & $1953(89,7 \%)$ & $3206(89,4 \%)$ & \\
\hline $\mathrm{Si}$ & $155(11,0 \%)$ & $224(10,3 \%)$ & $379(10,6 \%)$ & \\
\hline Sin dato & 0 & 0 & 0 & \\
\hline
\end{tabular}

Abreviaturas: TNEBD: Tumores neuroendocrinos bien diferenciados; CNEPD: Carcinomas neuroendocrinos pobremente diferenciados; THM: Tumores de histología mixta. 
El estadio del tumor se clasificó como localizado, regional, y con compromiso a distancia, según la clasificación de SEER. Como se describe en la versión 2018 del manual resumido de estadios proporcionado por SEER (https://seer. cancer.gov/tools/ssm/). Los tumores localizados se definieron como aquellos confinados al apéndice, los regionales se definieron como aquellos que comprometían por extensión directa la pared abdominal, grasa mesentérica o peri cólica, estructuras retroperitoneales, intestino delgado $\mathrm{u}$ otros órganos, o con compromiso tumoral en ganglios linfáticos regionales. Finalmente, aquellos con extensión a órganos distantes (glándulas suprarrenales, vejiga, diafragma, trompas de Falopio, piel, vesícula biliar, riñones, hígado, ovario, uréteres, útero, entre otros), a ganglios linfáticos distantes (mesentéricos inferiores o superiores) o con evidencia de carcinomatosis peritoneal, se definieron como tumores con compromiso a distancia.

\section{Análisis estadístico}

Utilizando el paquete dplyr disponible en $\mathrm{R}$ Studio $^{\circledR}$ (PBC, Boston, MA), los pacientes con TNE-A que cumplieron con los criterios de inclusión y exclusión fueron asignados aleatoriamente a un grupo de entrenamiento o de validación para construir y validar los nomogramas, respectivamente. Las variables cuantitativas fueron descritas como medianas con sus respectivos cuartiles 25 y 75, mientras que las variables cualitativas fueron descritas con su valor absoluto y relativo respecto al total del grupo.

Se utilizó la prueba de Chi cuadrado para comparar las diferencias en las características clínicas entre los dos grupos. La edad en el momento del diagnóstico fue analizada por el software X-tile con el fin de calcular los valores de corte en función de la información de supervivencia general. Se utilizó un análisis de regresión de riesgos proporcionales de Cox bivariado y multivariado con el software R Studio (versión 1.2.5042) para evaluar los factores pronósticos.

Las variables se calcularon mediante el cociente de riesgos instantáneos (HR) y el correspondiente intervalo de confianza del $95 \%$. Elegimos dos desenlaces principales: la supervivencia global y la supervivencia específica a cáncer. De acuerdo con los resultados del análisis de regresión Cox bivariado y multivariado, se construyeron los nomogramas tanto para supervivencia específica como general a 1, 2, 3 y 5 años con el paquete estadístico rms disponible en R (versión 3.5.3). Mientras tanto, se realizaron las validaciones internas y externas de los nomogramas pronósticos. Se utilizó el índice de concordancia de Harrell (índice $\mathrm{C}$ ) para evaluar la capacidad de discriminación de los mismos. Se construyeron curvas de calibración para comparar la consistencia entre las supervivencias predichas y observadas.

En esencia, el índice $C$ estima la probabilidad de que los resultados predichos sean consistentes con los resultados observados reales, similar a lo obtenido al evaluar el área bajo la curva ROC. El índice $C$ puede variar de 0,5 a 1,0, siendo clasificado un índice $C$ de 0,50 a 0,70 como de baja precisión, un índice $\mathrm{C}$ de 0,71 a 0,90 como de precisión media $\mathrm{y}$ finalmente aquellos con un valor mayor de 0,90 como de alta precisión ${ }^{9}$.

\section{Resultados}

El proceso de búsqueda en la base de datos de SEER permitió identificar 15.128 pacientes con diagnóstico de neoplasias del apéndice cecal. Posteriormente se aplicaron los criterios de inclusión y exclusión, siendo finalmente incluidos 3585 pacientes (figura 1).

\section{Análisis descriptivo}

Del total de 3585 individuos, fueron asignados 2177 pacientes en la cohorte de entrenamiento del modelo y 1408 pacientes en el grupo de validación. El grupo de entrenamiento se utilizó para la validación interna del modelo, mientras que el grupo de validación fue utilizado para la validación externa del mismo. En la cohorte de entrenamiento se reportaron 338 (15,5\%) fallecidos por cualquier causa, de los cuales 224 $(10,3 \%)$ tuvieron una causa relacionada a la neoplasia apendicular. Por otra parte, en la cohorte de validación fallecieron 229 (16,3\%) pacientes, de los cuales 155 (11,0\%) murieron por complicaciones asociadas a la neoplasia de estudio. Las características principales de las dos cohortes de 
estudio se encuentran resumidas en la tabla 1. Cabe destacar que no se presentaron diferencias significativas en ninguna variable evaluada entre los dos grupos de estudio.

Los tumores de histología mixta se presentaron en pacientes significativamente mayores y más frecuentemente en hombres, comparado con los otros subtipos histológicos de tumores neu- roendocrinos. Adicionalmente, los THM tendieron a ser de mayor tamaño y a afectar con mayor frecuencia estructuras regionales y distantes, siendo llevados más frecuentemente a procedimientos quirúrgicos de tipo colectomía y exhibiendo un mayor riesgo de mortalidad en comparación de sus contrapartes TNEBD y CNEPD (tabla 2 y figura 2).

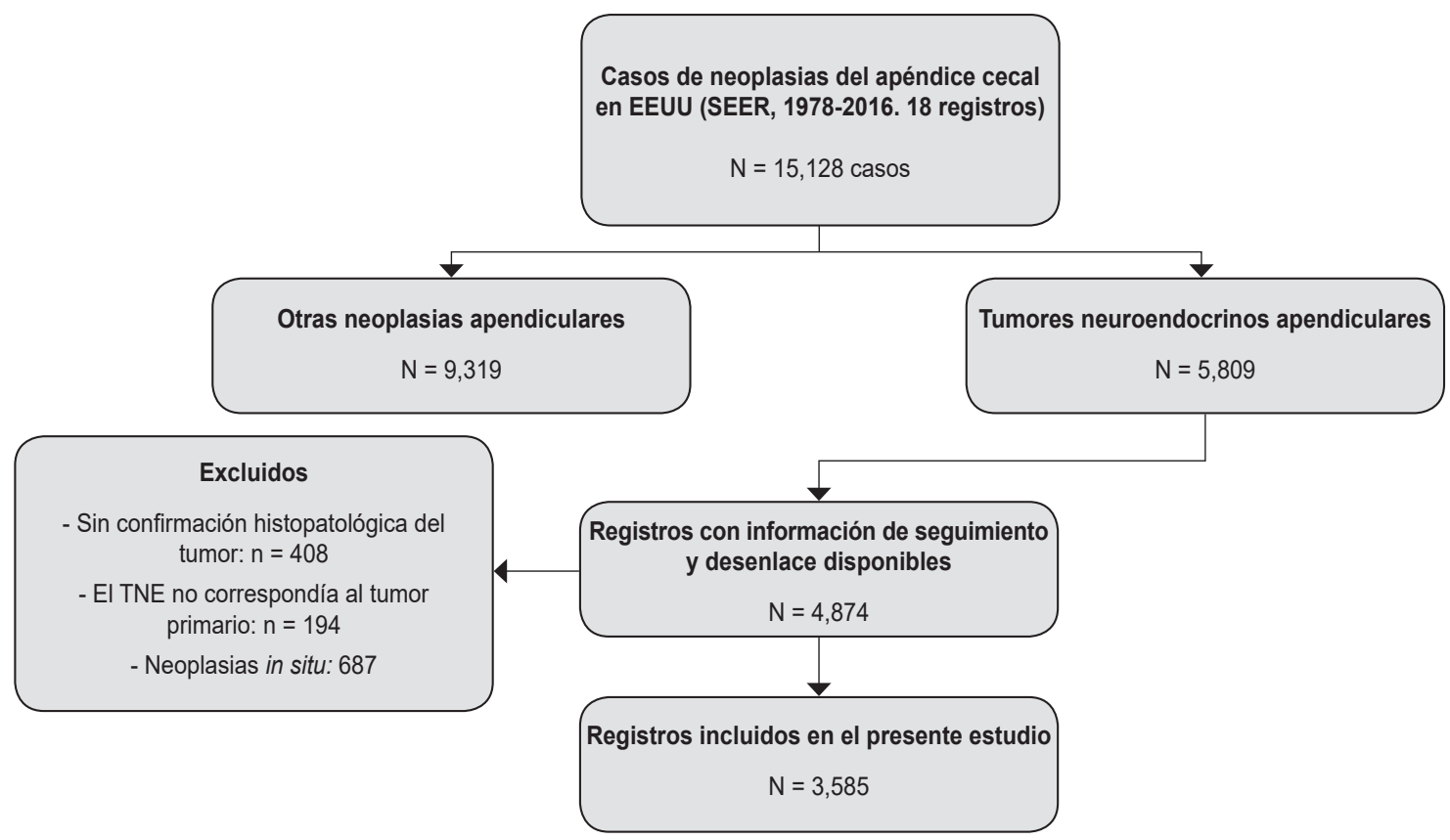

Figura 1. Diagrama de flujo que resume el proceso de selección de los casos de tumores neuroendocrinos del apéndice cecal en la base de datos de SEER.

Tabla 2. Características demográficas y clínicas de los pacientes con tumores neuroendocrinos apendiculares de acuerdo con el subtipo histológico.

\begin{tabular}{|c|c|c|c|c|c|}
\hline & $\begin{array}{c}\text { THM } \\
(n=1634)\end{array}$ & $\begin{array}{l}\text { CNEPD } \\
(n=183)\end{array}$ & $\begin{array}{l}\text { TNEBD } \\
(n=1322)\end{array}$ & $\begin{array}{c}\text { Total } \\
(n=3139)\end{array}$ & Valor de $p$ \\
\hline Edad & & & & & $<0,001$ \\
\hline Media (SD) & $55,1(13,0)$ & $41,3(17,0)$ & $39,5(17,9)$ & $47,7(17,3)$ & \\
\hline Mediana (Q1, Q3) & $55(47,6)$ & $40(27,53,5)$ & $37,5(25,53)$ & $50(35,60)$ & \\
\hline Mínima - Máxima & $8-91$ & $11-91$ & $4-94$ & $4-94$ & \\
\hline Sin datos & 0 & 0 & 0 & 0 & \\
\hline Edad (categorías) & & & & & $<0,001$ \\
\hline 0 - 52 años & $741(45,3 \%)$ & $137(74,9 \%)$ & 994 (75,2 \%) & $1872(59,6 \%)$ & \\
\hline 53 - 80 años & $843(51,6 \%)$ & $44(24 \%)$ & $314(23,8 \%)$ & $1201(38,3 \%)$ & \\
\hline 80 - 120 años & $50(3,1 \%)$ & $2(1,1 \%)$ & $14(1,1 \%)$ & $66(2,1 \%)$ & \\
\hline Sin dato & 0 & 0 & 0 & 0 & \\
\hline
\end{tabular}




\begin{tabular}{|c|c|c|c|c|c|}
\hline & $\begin{array}{c}\text { THM } \\
(n=1634)\end{array}$ & $\begin{array}{l}\text { CNEPD } \\
(n=183)\end{array}$ & $\begin{array}{c}\text { TNEBD } \\
(n=1322)\end{array}$ & $\begin{array}{c}\text { Total } \\
(n=3139)\end{array}$ & Valor de $p$ \\
\hline Sexo & & & & & $<0,001$ \\
\hline Mujer & $803(49,1 \%)$ & $107(58,5 \%)$ & $815(61,6 \%)$ & $1725(55 \%)$ & \\
\hline Hombre & $831(50,9 \%)$ & $76(41,5 \%)$ & $507(38,4 \%)$ & $1414(45 \%)$ & \\
\hline Sin dato & 0 & 0 & 0 & 0 & \\
\hline Raza & & & & & 0,501 \\
\hline Afrodescendiente & $145(8,9 \%)$ & $16(8,9 \%)$ & $93(7,2 \%)$ & $254(8,2 \%)$ & \\
\hline Blanco & $1415(87,0 \%)$ & $156(86,7 \%)$ & $1152(89,0 \%)$ & $2723(87,8 \%)$ & \\
\hline Otro & $66(4,1 \%)$ & $8(4,4 \%)$ & $49(3,8 \%)$ & $123(4 \%)$ & \\
\hline Sin dato & 8 & 3 & 28 & 39 & \\
\hline Tamaño tumoral & & & & & $<0,001$ \\
\hline$<1 \mathrm{~cm}$ & $137(16,3 \%)$ & $78(50,3 \%)$ & $716(64,3 \%)$ & $931(44,2 \%)$ & \\
\hline $1-2 \mathrm{~cm}$ & $208(24,8 \%)$ & $34(21,9 \%)$ & $244(21,9 \%)$ & $486(23,1 \%)$ & \\
\hline $2-5 \mathrm{~cm}$ & $317(37,8 \%)$ & $29(18,7 \%)$ & $133(11,9 \%)$ & $479(22,7 \%)$ & \\
\hline $5-10 \mathrm{~cm}$ & $168(20 \%)$ & $14(9 \%)$ & $18(1,6 \%)$ & $200(9,5 \%)$ & \\
\hline$>10 \mathrm{~cm}$ & $8(1 \%)$ & $0(0 \%)$ & $2(0,2 \%)$ & $10(0,5 \%)$ & \\
\hline Extensión & & & & & $<0,001$ \\
\hline Distante & $237(14,6 \%)$ & $18(9,8 \%)$ & $26(2 \%)$ & $281(9 \%)$ & \\
\hline Regional & $494(30,5 \%)$ & $42(23 \%)$ & $253(19,3 \%)$ & $789(25,3 \%)$ & \\
\hline Localizado & $890(54,9 \%)$ & $123(67,2 \%)$ & $1031(78,7$ \%) & $2044(65,6 \%)$ & \\
\hline Sin dato & 13 & 0 & 12 & 25 & \\
\hline Radioterapia & & & & & 0,085 \\
\hline No & $1625(99,4$ \%) & $182(99,5 \%)$ & $1321(99,9 \%)$ & $3128(99,6 \%)$ & \\
\hline $\mathrm{Si}$ & $9(0,6 \%)$ & $1(0,5 \%)$ & $1(0,1 \%)$ & $11(0,4 \%)$ & \\
\hline Sin dato & 0 & 0 & 0 & 0 & \\
\hline Quimioterapia & & & & & $<0,001$ \\
\hline No & $1276(78,1 \%)$ & $171(93,4 \%)$ & $1310(99,1 \%)$ & $2757(87,8 \%)$ & \\
\hline $\mathrm{Si}$ & $358(21,9 \%)$ & $12(6,6 \%)$ & $12(0,9 \%)$ & $382(12,2 \%)$ & \\
\hline Sin dato & 0 & 0 & 0 & 0 & \\
\hline Cirugía & & & & & $<0,001$ \\
\hline $\begin{array}{l}\text { Apendicectomía o } \\
\text { resección del ciego }\end{array}$ & $679(41,6 \%)$ & $133(72,7 \%)$ & $989(74,8 \%)$ & $1801(57,4 \%)$ & \\
\hline Colectomía & $955(58,4 \%)$ & $50(27,3 \%)$ & $333(25,2 \%)$ & $1338(42,6 \%)$ & \\
\hline Sin dato & 0 & 0 & 0 & 0 & \\
\hline Mortalidad global & & & & & $<0,001$ \\
\hline No & $1160(71 \%)$ & $165(90,2 \%)$ & $1289(97,5 \%)$ & $2614(83,3 \%)$ & \\
\hline $\mathrm{Si}$ & $474(29 \%)$ & $18(9,8 \%)$ & $33(2,5 \%)$ & $525(16,7 \%)$ & \\
\hline $\begin{array}{l}\text { Mortalidad asociada a } \\
\text { cáncer }\end{array}$ & & & & & $<0,001$ \\
\hline No & $1299(79,5 \%)$ & $170(92,9 \%)$ & $1309(99 \%)$ & $2778(88,5 \%)$ & \\
\hline $\mathrm{Si}$ & $335(20,5 \%)$ & $13(7,1 \%)$ & $13(1 \%)$ & $361(11,5 \%)$ & \\
\hline
\end{tabular}

*No se incluyeron los 3585 pacientes del análisis general, dado que en 446 pacientes no se reportaba el subtipo histológico. Abreviaturas: TNEBD: Tumores neuroendocrinos bien diferenciados; CNEPD: Carcinomas neuroendocrinos pobremente diferenciados; THM: Tumores de histología mixta. 


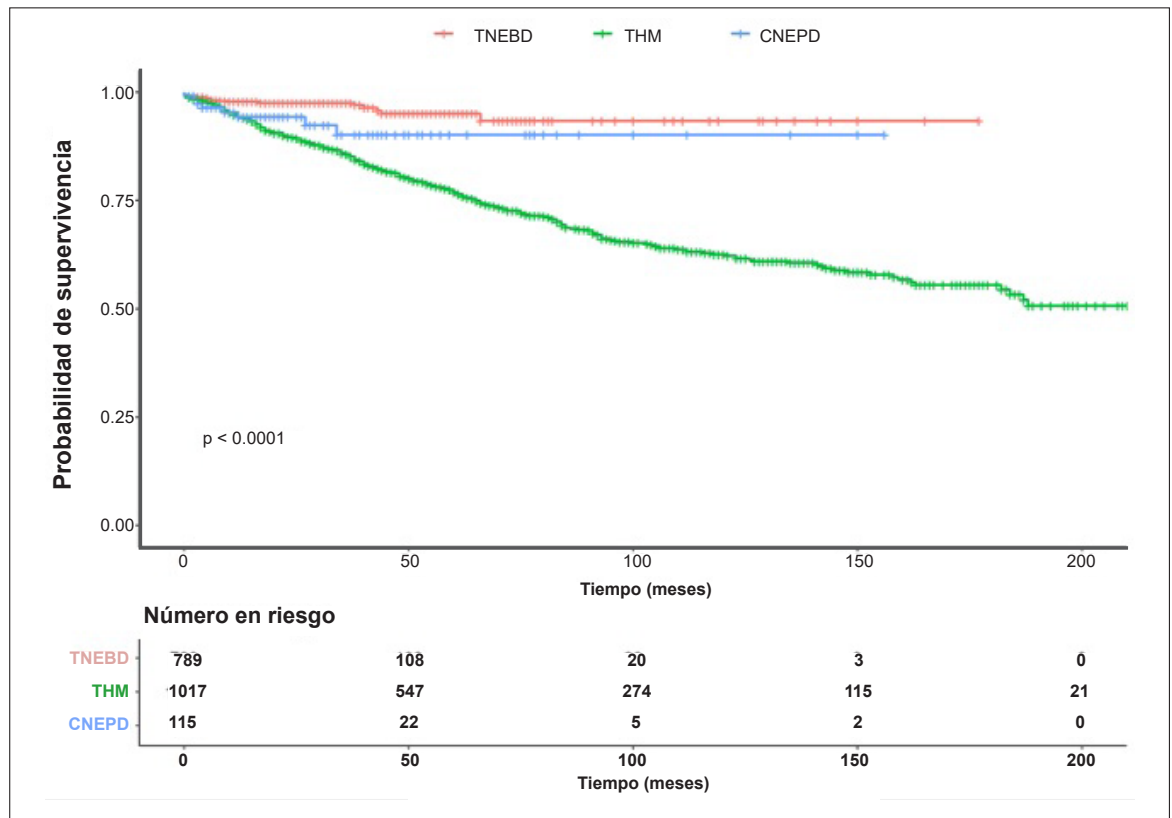

Figura 2. Gráfico de Kaplan-Meier que muestra la probabilidad de supervivencia en el seguimiento de los pacientes con tumores neuroendocrinos del apéndice cecal de acuerdo al subtipo histológico.

\section{Factores pronósticos para supervivencia global y específica de cáncer}

Múltiples variables se asociaron significativamente con el desenlace de sobrevida global en el análisis bivariado como se puede observar en la tabla 2 , sin embargo, solo la edad, la extensión, el subtipo histológico y el tamaño tumoral, fueron predictores independientes en el análisis multivariado. Se evidenció un mayor riesgo de mortalidad por cualquier causa a medida que se incrementaba la edad, la extensión y el tamaño tumoral. Por otra parte, el subtipo histológico mixto, se asoció con un riesgo significativamente mayor de mortalidad por cualquier causa, luego del ajuste multivariado (tabla 2).

De manera similar, la edad, extensión de la enfermedad, el tamaño tumoral y el subtipo histológico, fueron identificados como predictores independientes de supervivencia específica a cáncer, incluso después del análisis multivariado. El efecto por categoría fue similar al observado para supervivencia global (tabla 3 ).

\section{Construcción y validación del nomograma pronóstico}

Una vez se identificaron las variables predictoras independientes para sobrevida general y específica del cáncer, se procedió a crear los nomogramas pronósticos para estimar los valores de sobrevida a 1, 2, 3 y 5 años. En la figura 3 se pueden observar los nomogramas resultantes. Con el fin de contextualizar, el nomograma otorga a cada variable de pronóstico una puntuación en la escala de puntos de la parte superior. Al sumar estas puntuaciones se ubicará el valor en la puntuación total de la escala en la parte inferior, permitiendo finalmente predecir la supervivencia (ya sea general o específica al cáncer) trazando una línea vertical.

La validación interna de la cohorte de entrenamiento reveló un índice $\mathrm{C}$ de los nomogramas de supervivencia global y específica al cáncer de 0,80 $\left(\mathrm{IC}_{95 \%}: 0,70-0,76\right)$ y 0,87 (IC ${ }_{95 \%}$ : 0,73-0,81), respectivamente. Del mismo modo, el índice $C$ correspondiente en la cohorte de validación externa fue 0,81 (IC $\left.{ }_{95 \%}: 0,76-0,86\right)$ y 0,88 (IC $\left.{ }_{95 \%}: 0,83-0,92\right)$. 
Tabla 3. Análisis de regresión de Cox bivariado y multivariado de los factores asociados a la sobrevida global, en pacientes con tumores neuroendocrinos del apéndice cecal.

\begin{tabular}{|c|c|c|c|c|}
\hline Variables & Categorías Modalidades & $\mathbf{n}$ & HR univariado $\left(\mathrm{IC}_{95 \%}, \mathrm{p}\right)$ & HR multivariado $\left(\mathrm{IC}_{95 \%}, \mathrm{p}\right)$ \\
\hline \multirow{2}{*}{ Edad (categorías) } & 0 - 52 años & 1329 & \multicolumn{2}{|c|}{ Referencia } \\
\hline & 53 - 80 años & 806 & $2,7(2,1-3,3 p<0,001)$ & $1,8(1,2-2,5 p<0,001)$ \\
\hline \multirow{4}{*}{ Raza } & 80 - 120 años & 42 & $9,8(6,3-15,5 p<0,001)$ & $6,3(3,0-12,8 p<0,001)$ \\
\hline & Afrodescendiente & 181 & \multicolumn{2}{|c|}{ Referencia } \\
\hline & Blanco & 1882 & $0,6(0,4-0,9 p=0.021)$ & $6,1(3,0-12,6 p=0,691)$ \\
\hline & Otro & 88 & $0,7(0,4-1,4 p=0,461)$ & $0,61(0,1-1,8 p=0,348)$ \\
\hline \multirow{2}{*}{ Sexo } & Femenino & 1230 & \multicolumn{2}{|c|}{ Referencia } \\
\hline & Masculino & 947 & $1,0(0,8-1,3 p=0,547)$ & \\
\hline \multirow[t]{2}{*}{ Estado marital } & Casado & 1096 & \multicolumn{2}{|c|}{ Referencia } \\
\hline & Soltero & 953 & $1,0(0,8-1,3 p=0,458)$ & \\
\hline \multirow[t]{2}{*}{ Grado } & Grado I-II & 1022 & \multicolumn{2}{|c|}{ Referencia } \\
\hline & Grado III-IV & 260 & $1,1(0,7-1,7 p=0,570)$ & \\
\hline \multirow{3}{*}{ Subtipo histológico } & THM & 1017 & \multicolumn{2}{|c|}{ Referencia } \\
\hline & TNEBD & 789 & $0,4(0,1-0,3 p<0,001)$ & $0,4(0,2-0,6 p<0,001)$ \\
\hline & CNEPD & 115 & $0,4(0,2-0,9 p=0,049)$ & $0,4(0,1-0,9 p=0.046)$ \\
\hline \multirow{4}{*}{ Extensión } & Distante & 178 & \multicolumn{2}{|c|}{ Referencia } \\
\hline & Regional & 546 & $0,1(0,1-0,1 p<0,001)$ & $0,1(0,1-0,2 p<0,001)$ \\
\hline & Localizado & 1431 & $0,0(0,0-0,1 p<0,001)$ & $0,1(0,0-0,1 p<0,001)$ \\
\hline & $<1 \mathrm{~cm}$ & 429 & \multicolumn{2}{|c|}{ Referencia } \\
\hline \multirow[t]{4}{*}{ Tamaño del tumor } & $1-2 \mathrm{~cm}$ & 240 & $1,4(0,8-2,5 p=0,139)$ & $0,8(0,4-1,5 p=0,581)$ \\
\hline & $2-5 \mathrm{~cm}$ & 524 & $3,2(2,0-5,1 p<0,001)$ & $1,1(0,6-2,0 p=0,646)$ \\
\hline & $5-10 \mathrm{~cm}$ & 77 & $5,5(3,3-9,1 p<0,001)$ & $1,2(0,6-2,3 p=0,484)$ \\
\hline & $>10 \mathrm{~cm}$ & 7 & $22,9(6,9-76,0 p<0,001)$ & $7,7(2,1-28,2 p<0,001)$ \\
\hline \multirow[t]{2}{*}{ Quimioterapia } & No & 1930 & \multicolumn{2}{|c|}{ Referencia } \\
\hline & $\mathrm{Si}$ & 247 & $4,4(3,5-5,5 p<0,001)$ & $1,2(0,7-1,9 p=0,382)$ \\
\hline \multirow[t]{2}{*}{ Radioterapia } & No & 2170 & \multicolumn{2}{|c|}{ Referencia } \\
\hline & $\mathrm{Si}$ & 7 & $6,2(2,6-15,2 p<0,001)$ & $2,8(0,6-12,1 p=0,164)$ \\
\hline \multirow[t]{2}{*}{ Cirugía } & $\begin{array}{l}\text { Apendicectomía o } \\
\text { resección del ciego }\end{array}$ & 1263 & \multicolumn{2}{|c|}{ Referencia } \\
\hline & Colectomía & 914 & $1,4(1,1-1,7 p=0,002)$ & $1,1(0,8-1,6 p=0,452)$ \\
\hline
\end{tabular}

*No se incluye el total de pacientes del análisis general, dado que hubo individuos sin datos reportados en algunas variables. El número de individuos con datos faltantes por variable se puede encontrar en la Tabla 1.

Abreviaturas: TNEBD: Tumores neuroendocrinos bien diferenciados; CNEPD: Carcinomas neuroendocrinos pobremente diferenciados; THM: Tumores de histología mixta. 
A.

Puntos

Edad (categoría)

Extensión

Tamaño tumoral

Subtipo histológico

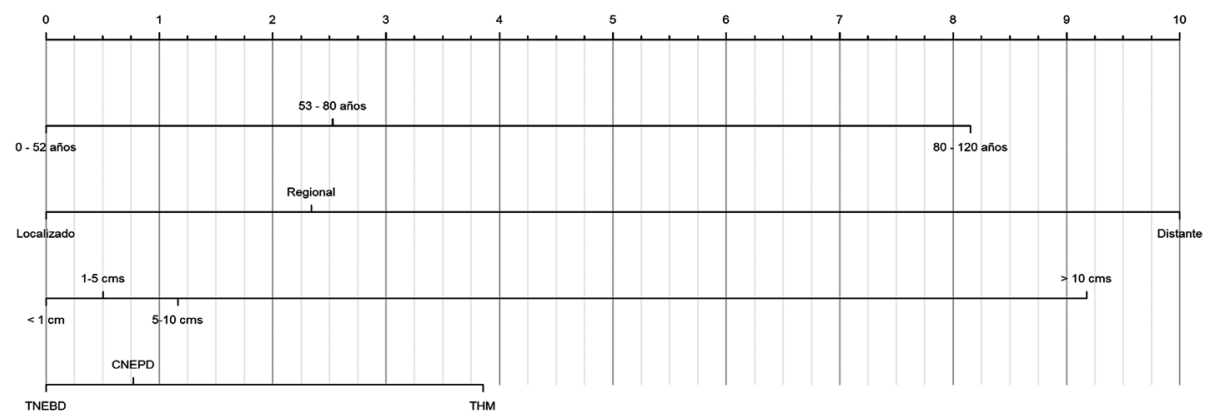

Puntos totales

Supervivencia a 1 año

Supervivencia a 2 años

Supervivencia a 3 años

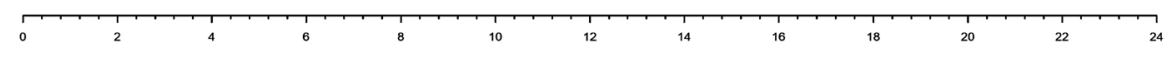

Supervivencia a 5 años

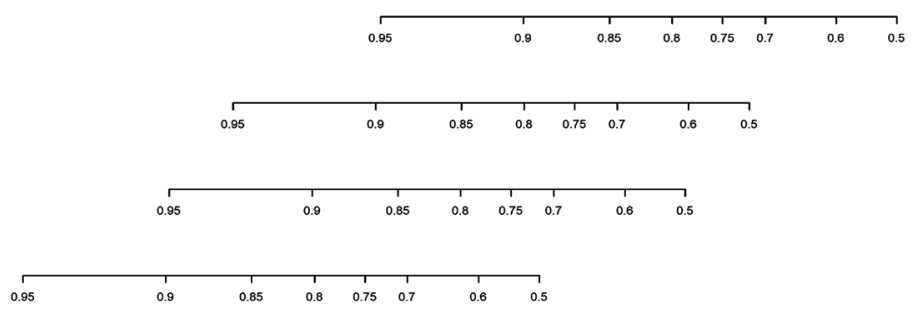

B.

Puntos

Edad (categoría)

Extensión

Tamaño tumoral

Subtipo histológico

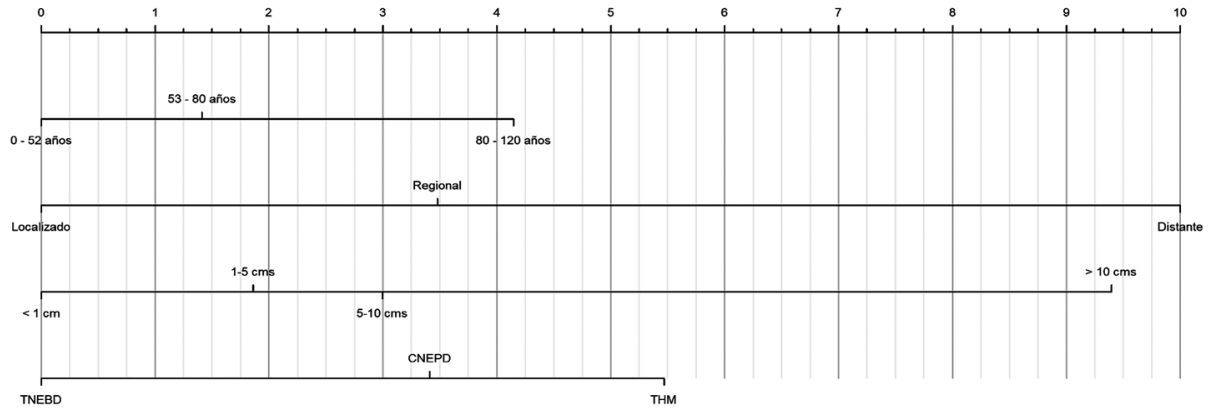

Puntos totales

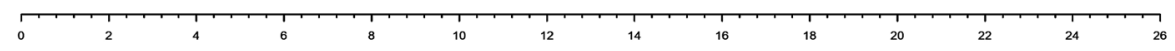

Supervivencia a 1 año

Supervivencia a 2 años

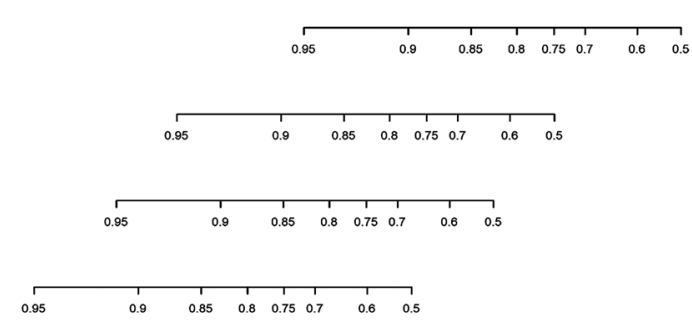

Supervivencia a 3 años

Supervivencia a 5 años

Figura 3. Nomogramas de predicción de sobrevida general (Panel A) y específica a cáncer (Panel B) a 1, 2, 3 y 5 años en tumores neuroendocrinos apendiculares (TNE-A). Notas: Los puntos de cada variable se obtienen trazando una línea vertical entre cada variable y la escala de puntos (superior). La tasa de supervivencia prevista se obtiene sumando los puntos obtenidos por variable y con los puntos totales obtenidos se traza una línea vertical desde la escala de puntos totales hasta la de supervivencia general de cada año.

TNEBD: Tumores neuroendocrinos bien diferenciados; CNEPD: Carcinomas neuroendocrinos pobremente diferenciados; THM: Tumores de histología mixta. 
Tabla 4. Análisis de regresión de Cox bivariado y multivariado de los factores asociados a la supervivencia específica para cáncer, en pacientes con tumores neuroendocrinos del apéndice cecal.

\begin{tabular}{|c|c|c|c|c|}
\hline Variables & $\begin{array}{l}\text { Categorías } \\
\text { Modalidades }\end{array}$ & $\mathbf{n}$ & $\begin{array}{l}\text { HR univariado } \\
\qquad\left(\mathrm{IC}_{95 \%}, \mathbf{p}\right)\end{array}$ & $\begin{array}{l}\text { HR multivariado } \\
\qquad\left(\mathrm{IC}_{95 \%}, \mathrm{p}\right)\end{array}$ \\
\hline \multirow[t]{3}{*}{ Edad (categoría) } & 0 - 52 años & 1329 & Referencia & \\
\hline & 53 - 80 años & 806 & $2,2(1,7-3,0 p<0,001)$ & $1,4(1,1-2,4 p=0,005)$ \\
\hline & 80 - 120 años & 42 & $5,3(2,7-10,3 p<0,001)$ & $2,6(1,7-8,6 p<0,001)$ \\
\hline \multirow[t]{3}{*}{ Raza } & Afrodescendiente & 181 & Referencia & \\
\hline & Otro & 88 & $0,7(0,3-1,5 p=0,492)$ & $0,6(0,1-1,9 p=0,414)$ \\
\hline & Blanco & 1882 & $0,58(0,4-0,8 p=0,005)$ & $0,6(0,3-1,2 p=0,193)$ \\
\hline \multirow[t]{2}{*}{ Sexo } & Femenino & 1230 & Referencia & \\
\hline & Masculino & 947 & $0,8(0,6-1,0 p=0,192)$ & \\
\hline \multirow[t]{2}{*}{ Estado marital } & Casado & 1096 & Referencia & \\
\hline & Soltero & 953 & $0,8(0,6-1,1 p=0,216)$ & \\
\hline \multirow[t]{2}{*}{ Grado } & Grado I-II & 1022 & Referencia & \\
\hline & Grado III-IV & 260 & $1,33(0,8-2,1 p=0,232)$ & \\
\hline \multirow[t]{3}{*}{ Subtipo histológico } & THM & 1017 & Referencia & \\
\hline & TNEBD & 789 & $8,9(4,3-18,2 p<0,001)$ & $0,1(0,0-0,4 p<0,001)$ \\
\hline & CNEPD & 115 & $4,9(1,8-13,7 p=0,002)$ & $0,5(0,2-1,3 p=0,186)$ \\
\hline \multirow[t]{3}{*}{ Extensión } & Distante & 178 & Referencia & \\
\hline & Localizado & 1431 & $0,0(0,0-0,0 p<0,001)$ & $0,0(0,0-0,0 p<0.001)$ \\
\hline & Regional & 546 & $0,1(0,0-0,1 p<0,001)$ & $0,1(0,0-0,2 p<0,001)$ \\
\hline \multirow[t]{5}{*}{ Tamaño del tumor } & $<1 \mathrm{~cm}$ & 682 & Referencia & \\
\hline & $1-2 \mathrm{~cm}$ & 240 & $2,5(1,0-6,2 p=0,04)$ & $1,1(0,4-3,0 p=0,385)$ \\
\hline & $2-5 \mathrm{~cm}$ & 524 & $8,8(4,0-19,6 p<0,001)$ & $2,1(0,8-5,3 p=0,199)$ \\
\hline & $5-10 \mathrm{~cm}$ & 133 & $19,1(8,5-43,2 p<0,001)$ & $2,6(0,9-6,8 p=0,055)$ \\
\hline & $>10 \mathrm{~cm}$ & 4 & $80,0(20,6-310,4 p<0,001)$ & $17,8(4,0-78,1 p<0,001)$ \\
\hline \multirow[t]{2}{*}{ Quimioterapia } & No & 1930 & Referencia & \\
\hline & Yes & 247 & $7,5(5,8-9,8 p<0,001)$ & $1,2(0,7-2,1 p=0,367)$ \\
\hline \multirow[t]{2}{*}{ Radioterapia } & No & 2170 & Referencia & \\
\hline & Yes & 7 & $9,3(3,8-22,7 p<0,001)$ & $2,5(0,7-2,1 p=0,212)$ \\
\hline \multirow[t]{2}{*}{ Cirugía } & $\begin{array}{l}\text { Apendicectomía o } \\
\text { resección del ciego }\end{array}$ & 1263 & Referencia & \\
\hline & Colectomía & 914 & $1,7(1,3-2,2 p<0,001)$ & $1,1(0,7-1,7 p=0,561)$ \\
\hline
\end{tabular}

*No se incluye el total de pacientes del análisis general, dado que hubo individuos sin datos reportados en algunas variables. El número de individuos con datos faltantes por variable se puede encontrar en la Tabla 1.

Abreviaturas: TNEBD: Tumores neuroendocrinos bien diferenciados; CNEPD: Carcinomas neuroendocrinos pobremente diferenciados; THM: Tumores de histología mixta.

Estos resultados confirman que nuestros nomogramas de pronóstico son razonablemente precisos. El proceso de calibración (figura 4) muestra que la tasa de supervivencia real, concuerda bien con la predicción del nomograma.

\section{Discusión}

Los tumores neuroendocrinos del apéndice cecal (TNE-A) corresponden a un conjunto de neoplasias de comportamiento heterogéneo, susceptibles de tratamiento, con resultados más favorables 
y pronóstico excelente, si se comparan con TNE de otras localizaciones ${ }^{10}$. Sin embargo, la gran variedad de subtipos de TNE-A, hace necesario un abordaje individualizado que permita orientar las estrategias terapéuticas teniendo en cuenta un pronóstico estimado.

Este estudio propone un nomograma pronóstico de sobrevida para pacientes con TNE-A, teniendo en cuenta su subtipo histológico, y encontró que la edad, la extensión de la enfermedad, el tamaño tumoral y el subtipo histológico, son factores pronóstico independientes para la supervivencia de estos pacientes. Se presentan los nomogramas con estos factores, encontrando un índice-C para mortalidad global de 0,81 y para mortalidad específica por cáncer de 0,88 , lo cual pone en evidencia el buen rendimiento de los mismos para la discriminación de estos desenlaces.

En el presente estudio, la edad al momento del diagnóstico fue un factor pronóstico independiente de mortalidad global y específica, asociándose una edad mayor a 80 años, con un peor pronóstico en los pacientes con tumores neuroendocrinos del apéndice.

Para el subtipo histológico, utilizamos la última clasificación de TNE-A de la OMS ${ }^{11}$. Al igual que en nuestro estudio, investigaciones publicadas en 2011 y 2017 demostraron que, además del tamaño tumoral, el subtipo histológico es un factor deter- minante del riesgo de metástasis ganglionar ${ }^{12,13}$. Aunque previamente se consideraba $20 \mathrm{~mm}$ como punto de corte para definir un alto riesgo de metástasis ganglionar, Sarshekeh et al. observaron que los pacientes con TNEBD y THM tuvieron mayor compromiso ganglionar regional cuando su tamaño era mayor de $10 \mathrm{~mm}$, proponiendo este tamaño como nuevo punto de corte para predecir el riesgo de este desenlace ${ }^{13}$.

En el presente estudio no se encontraron diferencias significativas cuando se comparó la sobrevida de aquellos pacientes con tumores menores de $1 \mathrm{~cm}$ con aquellos de entre $1 \mathrm{y} 2 \mathrm{~cm}$. Al ajustar por los demás factores independientes, se evidenció un mayor impacto en el pronóstico de los pacientes con tamaño tumoral mayor de $10 \mathrm{~cm}$.

El subtipo histológico más identificado en nuestro estudio fue el THM, el cual ocurrió en adultos de mayor edad, con mayor tamaño tumoral y con un porcentaje más alto de extensión regional y distante, requiriendo más comúnmente hemicolectomía y mostrando una supervivencia significativamente peor en comparación con los demás subtipos. Sarshekeh et al. también evidenciaron que el THM correspondía al subtipo histológico más frecuente, sin embargo, encontraron que los CNEPD tuvieron el peor pronóstico, esto puede ser debido al limitado número de pacientes con este subtipo histológico incluido dentro de su
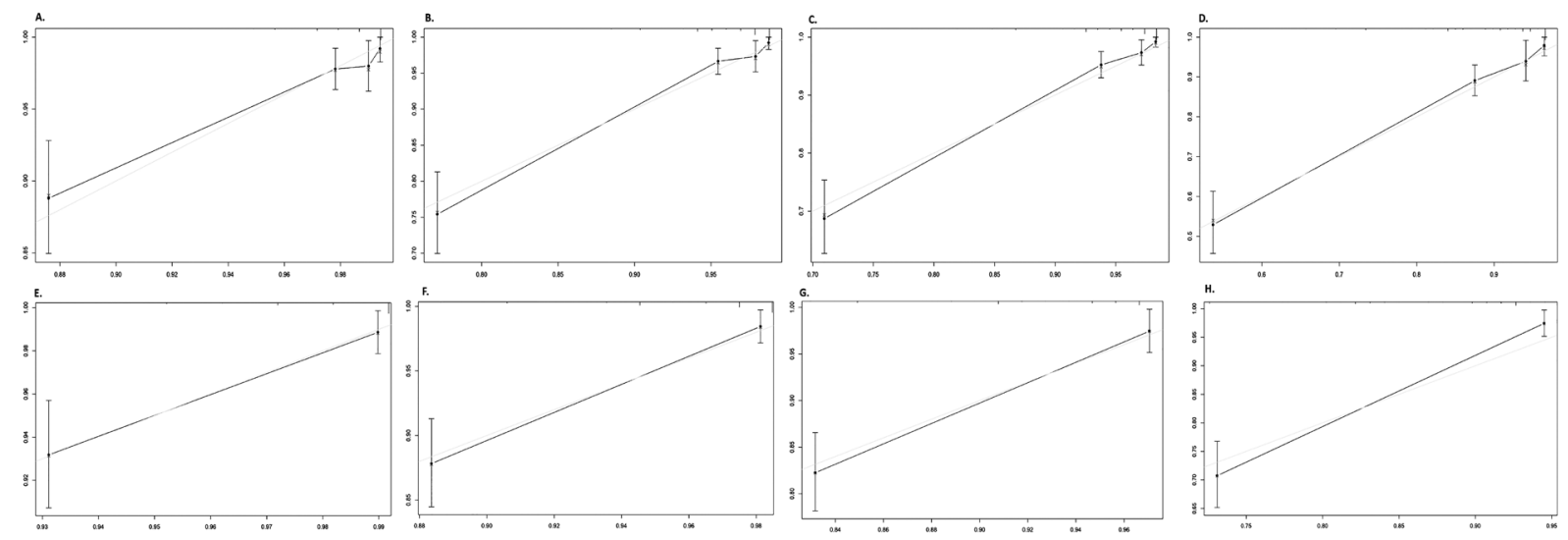

Figura 4. Curvas de calibración del nomograma de supervivencia general a 1 año (A), 2 años (B), 3 años (C) y 5 años (D); Curvas de calibración del nomograma de supervivencia específica al cáncer a 1 año (E), 2 años (F), 3 años (G) y 5 años $(H)$. Notas: La línea discontinua representa una coincidencia excelente entre el resultado de supervivencia real (eje $Y$ ) y la predicción del nomograma (eje X). Una distancia más cercana entre la línea discontinua y los puntos indican una mayor precisión de predicción. 
análisis (132). De forma llamativa, encontraron una mayor tasa de compromiso metastásico ganglionar en los pacientes con TNEBD, sin embargo, este subtipo histológico se asoció, al igual que en nuestro estudio y en estudios previos, a un pronóstico más favorable ${ }^{12,14,15}$.

$\mathrm{Al}$ igual que en estudios previos, en este grupo de pacientes, el manejo quirúrgico (apendicectomía vs hemicolectomía) no demostró ser un factor pronóstico independiente de mortalidad al realizar el ajuste por edad, extensión, tamaño tumoral y tipo histológico. Esto se puede deber a la existencia de claros lineamientos por consensos internacionales en los cuales se especifica la extensión del manejo quirúrgico según el tamaño tumoral, el compromiso de la base y de los márgenes de resección y de acuerdo con la profundidad de invasión del meso del apéndice ${ }^{16,17,18}$.

En los pacientes llevados a hemicolectomía, se encontró que el tamaño y la extensión tumoral fueron significativamente mayores, que en aquellos a quienes se les realizó apendicectomía, siendo más frecuente la hemicolectomía en los pacientes con THM. Por tanto, el resultado del pronóstico similar en los pacientes manejados con colectomía o con apendicectomía, podría derivar de estas diferencias. Sin embargo, se debe tener en cuenta como probable objeto de confusión que la base de datos SEER de la cual se obtuvieron las cifras para este análisis, incluye las resecciones de segmentarias de colon, de ciego, de íleon, o sus combinaciones con el mismo código de registro de las apendicectomías.

El único nomograma realizado previamente para TNE-A, fue desarrollado por Mosquera et al. en $2017^{2}$. El objetivo fue predecir el riesgo de metástasis ganglionares, teniendo en cuenta el tamaño tumoral y la profundidad de la invasión. Se encontró una asociación entre el tamaño tumoral, la profundidad de invasión y el manejo quirúrgico, con la incidencia de metástasis ganglionares, obteniendo un rendimiento aceptable (AUC 0,89). En dicho estudio, se excluyeron los TNE de alto grado y los pacientes menores de 18 años.

Los hallazgos del presente nomograma, complementa los resultados del estudio previo, dado que, más allá de evaluar solo el riesgo de progre- sión tumoral, permite predecir la supervivencia de los pacientes.

\section{Fortalezas y limitaciones}

El análisis de casos provenientes de un registro nacional como SEER (el cual goza de una solidez metodológica y logística reconocida) facilitó la extrapolación de los resultados obtenidos, dado el amplio número de casos y su origen como datos de la vida real. Lo anterior, permitió además realizar un ajuste por subtipo histológico, utilizando los códigos de histología de SEER de acuerdo con la clasificación más reciente de la OMS de tumores apendiculares, por lo que se evaluó con mayor claridad el comportamiento de estos tumores considerando su heterogeneidad.

Este estudio tuvo limitaciones, principalmente derivadas de su carácter retrospectivo y de la falta de datos clínicos, información sobre la recurrencia de la enfermedad y el tipo de quimioterapia utilizada, entre otros, lo cual limita de manera importante la evaluación de los factores asociados a la sobrevida. Existe la posibilidad de que, al ser considerados habitualmente como benignos, una proporción importante de TNE-A no haya sido reportada adecuadamente en la base de datos del programa SEER.

Finalmente, la clasificación de los procedimientos también resulta una limitación importante, considerando que la base de datos de SEER utiliza el mismo código para procedimientos como apendicectomías, resecciones de ciego, y colectomías parciales. De manera similar, las colectomías (hemicolectomías o colectomías totales) fueron codificadas de manera idéntica en la base de datos, por lo que, en el presente estudio, cualquiera de estos dos procedimientos se clasificó como "colectomía".

\section{Conclusión}

El presente estudio identificó a la edad, el tamaño tumoral, el subtipo histológico y la extensión de la enfermedad como los únicos predictores independientes de sobrevida global y específica a cáncer en pacientes con tumores neuroendocrinos del apéndice cecal. A partir de estos se construyó un nomograma práctico y con una capacidad de discriminación sobresaliente para la predicción de estos desenlaces. De manera interesante, los 
procedimientos de tipo colectomía no presentaron una ventaja respecto a la sobrevida de estos pacientes a comparación de la apendicectomía/ resección de ciego. Se requieren más estudios para evaluar con mayor claridad el rol de los distintos procedimientos quirúrgicos en la sobrevida de estos pacientes, y la utilidad de las distintas modalidades adyuvantes en estos contextos.

\section{Cumplimiento de normas éticas}

Consentimiento informado: El presente estudio no requirió consentimiento informado ni aprobación por parte de un comité de ética dado que se basa en datos extraídos de una base de datos de acceso libre. Los autores cuentan con un usuario autorizado para el acceso a la información, y se firmaron los acuerdos de manejo de datos correspondientes.

Conflicto de interés: los autores declaran no tener conflictos de interés.

Fuentes de financiación: Los recursos de financiación de este proyecto de investigación, provienen en su totalidad de aportes de los autores.

\section{Contribución de los autores:}

Concepción y diseño del estudio: Andrea Carolina Quiroga-Centeno, Carlos Augusto Quiroga-Centeno, Juan Paulo Serrano-Pastrana, Sergio Alejandro Gómez-Ochoa. Adquisición de datos: Andrea Carolina Quiroga-Centeno, Sergio Alejandro Gómez-Ochoa.

Análisis e interpretación de datos: Andrea Carolina Quiroga-Centeno, Sergio Alejandro Gómez-Ochoa.

Redacción del manuscrito: Andrea Carolina QuirogaCenteno, Carlos Augusto Quiroga-Centeno, Juan Paulo Serrano-Pastrana, Sergio Alejandro Gómez-Ochoa.

Revisión crítica: Andrea Carolina Quiroga-Centeno, Carlos Augusto Quiroga-Centeno, Juan Paulo SerranoPastrana, Sergio Alejandro Gómez-Ochoa.

\section{Referencias}

1. Moertel CG, Weiland LH, Nagorney DM, Dockerty MB. Carcinoid tumor of the appendix: Treatment and prognosis. N Engl J Med. 1987;317:1699-701. https://doi.org/10.1056/NEJM198712313172704

2. Mosquera C, Fitzgerald TL, Vora H, Grzybowski M. Novel nomogram combining depth of invasion and size can accurately predict the risk for regional nodal metastases for appendiceal neuroendocrine tumors (A-NET). J Surg Oncol. 2017;116:651-7. https://doi.org/10.1002/jso.24714
3. Pinto MP, Muñoz Medel M, Carrillo D, Retamal IN, Bravo ML, Valenzuela Y, et al. Chilean registry for neuroendocrine tumors: A Latin American perspective. Horm Cancer. 2019;10:3-10. https://doi.org/10.1007/s12672-018-0354-5

4. Dasari A, Shen C, Halperin D, Zhao B, Zhou S, Xu Y, et al. Trends in the incidence, prevalence, and survival outcomes in patients with neuroendocrine tumors in the United States. JAMA Oncol. 2017;3:1335. https://doi.org/10.1001/jamaoncol.2017.0589

5. Abdelaal A, El Ansari W, Al-Bozom I, Khawar M, Shahid F, Aleter A, et al. Frequency, characteristics and outcomes of appendicular neuroendocrine tumors: A cross-sectional study from an academic tertiary care hospital. Ann Med Surg. 2017;21:20-4. https://doi.org/10.1016/j.amsu.2017.07.043

6. Galanopoulos M, Toumpanakis C. The problem of appendiceal carcinoids. Endocrinol Metab Clin North Am. 2018;47:661-9. https://doi.org/10.1016/j.ecl.2018.04.004

7. Taggart MW, Abraham SC, Overman MJ, Mansfield PF, Rashid A. Goblet cell carcinoid tumor, mixed goblet cell carcinoid-adenocarcinoma, and adenocarcinoma of the appendix: comparison of clinicopathologic features and prognosis. Arch Pathol Lab Med. 2015;139:782-90. https://doi.org/10.5858/arpa.2013-0047-OA

8. Balachandran VP, Gonen M, Smith JJ, DeMatteo RP. Nomograms in oncology: more than meets the eye. Lancet Oncol. 2015;16:e173-80. https://doi.org/10.1016/S1470-2045(14)71116-7

9. Vickers AJ, Cronin AM. Everything you always wanted to know about evaluating prediction models (but were too afraid to ask). Urology. 2010;76:1298. https://doi.org/10.1016/j.urology.2010.06.019

10. Amr B, Froghi F, Edmond M, Haq K, Thengungal Kochupapy R. Management and outcomes of appendicular neuroendocrine tumours: Retrospective review with 5-year follow-up. Eur J Surg Oncol EJSO. 2015;41:1243-6. https://doi.org/10.1016/j.ejso.2015.06.010

11. Rindi G, Arnold R, Bosman FT, Capella FT, Klimstra DS, Kloppel G, et al. Nomenclature and classification of neuroendocrine neoplasms of the digestive system. In: WHO Classification of Tumours of the Digestive System, Bosman TF, Hruban RH, Theise ND. (Eds). International Agency for Research on Cancer (IARC); Lyon. 2010.

12. Groth SS, Virnig BA, Al-Refaie WB, Jarosek SL, Jensen EH, Tuttle TM. Appendiceal carcinoid tumors: Predictors of lymph node metastasis and the impact of right hemicolectomy on survival. J Surg Oncol. 2011;103:3945. https://doi.org/10.1002/jso.21764

13. Sarshekeh AM, Advani S, Halperin DM, Conrad C, Shen $\mathrm{C}$, Yao JC, et al. Regional lymph node involvement and outcomes in appendiceal neuroendocrine tumors: a SEER database analysis. Oncotarget. 2017;8:99541-51. https://doi.org/10.18632/oncotarget.20362 
14. Ciarrocchi A, Pietroletti R, Carlei F, Necozione S, Amicucci G. Propensity adjusted appraisal of the surgical strategy for appendiceal carcinoids. Tech Coloproctology. 2015;19:35-41.

https://doi.org/10.1007/s10151-014-1249-2

15. Mullen JT, Savarese DMF. Carcinoid tumors of the appendix: A population-based study: Carcinoid Tumors of the Appendix. J Surg Oncol. 2011;104:41-4. https://doi.org/10.1002/jso.21888

16. Pape U-F, Perren A, Niederle B, Gross D, Gress T, Costa F, et al. ENETS Consensus guidelines for the management of patients with neuroendocrine neoplasms from the jejuno-ileum and the appendix including goblet cell carcinomas. Neuroendocrinology. 2012;95(2):135-56. https://doi.org/10.1159/000335629

17. Kunz PL, Reidy-Lagunes D, Anthony LB, Bertino EM, Brendtro K, Chan JA, et al. Consensus guidelines for the management and treatment of neuroendocrine tumors. Pancreas. 2013;42:557-77.

https://doi.org/10.1097/MPA.0b013e31828e34a4

18. National Comprehensive Cancer Network. NCCN clinical practice guidelines in oncology: neuroendocrine tumors, Version 2.2016. Disponible en: http://www. nccn.org/professionals/physician_gls/pdf/neuroendocrine.pdf 